\title{
SAÚDE SEXUAL E ENVELHECIMENTO: REVISÃO DA LITERATURA E APONTAMENTOS PARA A EDUCAÇÃO SEXUAL
}

\author{
SALUD SEXUAL Y ENVEJECIMENTO: REVISIÓN DE LA LITERATURA Y \\ APUNTES PARA LA EDUCACIÓN SEXUAL
}

\author{
SEXUAL HEALTH AND AGING: LITERATURE REVIEW AND NOTES FOR SEX \\ EDUCATION
}

\author{
Ana Cláudia BORTOLOZZI ${ }^{1}$ \\ Tatiana de Cássia Ramos NETTO
}

RESUMO: A expectativa de vida aumentou no Brasil e o envelhecimento, como um fenômeno biológico e psicossocial, é uma fase de atenção de profissionais e pesquisadores. Embora haja preconceitos sobre a sexualidade no envelhecimento, muitos idosos têm vida sexual ativa e são vulneráveis às infecções sexualmente transmissíveis. Esta pesquisa documental realizou uma revisão da literatura sobre saúde sexual e envelhecimento, selecionando 68 artigos para análise de conteúdo. Os artigos foram encontrados entre 2001 e 2018, sendo a maior parte em 2015 e nas áreas da Enfermagem, Saúde Coletiva e Gerontologia. Os resultados foram descritos nas categorias: (A) Violência contra idosos (as); (B) Doenças e problemas físicos no envelhecimento; (C) Saúde sexual e sexualidade; (D) Vivências de idosos com HIV/Aids. (E) HIV/Aids e idosos: vulnerabilidade e prevenção. Conclui-se a necessidade da educação sexual para diminuir o nível de vulnerabilidade de pessoas idosas sobre a saúde sexual.

PALAVRAS-CHAVE: Envelhecimento. Saúde Sexual. Educação Sexual.

RESUMÉN: La expectativa de vida aumentó en Brasil y el envejecimiento, como un fenómeno biológico y psicosocial, es una fase que necesita de atención de profesionales e investigadores. Aunque hay preconceptos sobre la sexualidad en el envejecimiento, muchos ancianos tienen vida sexual activa y son vulnerables a las infecciones de transmisión sexual. Esta investigación documental realizó una revisión de la literatura sobre salud sexual y envejecimiento, seleccionando 68 artículos para análisis de contenido. Los artículos fueron encontrados entre 2001 y 2018, siendo la mayor parte en 2015 y en las áreas de la Enfermería, Salud Colectiva $y$ Gerontología. Los resultados se describen en las categorías: (A) Violencia contra los ancianos (as); (B) Enfermedades y problemas fisicos en el envejecimiento; (C) Salud sexual y sexualidad; (D) Vivencias de ancianos con VIH / SIDA. (E) VIH / SIDA y ancianos: vulnerabilidad y prevención.

PALABRAS CLAVE: Envejecimiento. Salud Sexual. Educación sexual.

\footnotetext{
${ }^{1}$ Universidade Estadual Paulista (UNESP), Bauru - SP - Brasil. Docente no Departamento de Psicologia. Pósdoutorado em Educação (UMINHO) - Portugal. Associada em Inclusão, Educação Sexual e Desenvolvimento Humano. ORCID: https://orcid.org/0000-0003-4796-5451. E-mail: claudia.bortolozzi@unesp.br

${ }^{2}$ Universidade Estadual Paulista (UNESP), Bauru - SP, Brasil. Doutoranda no programa de Pós-graduação em Psicologia do Desenvolvimento e Aprendizagem. ORCID: https://orcid.org/0000-0002-9907-790X. E-mail: taty_psy@yahoo.com.br
}

RIAEE - Revista Ibero-Americana de Estudos em Educação, Araraquara, v. 15, n. esp. 4, p. 2699-2712, dez., 2020. e-ISSN: 1982-5587 
ABSTRACT: Life expectancy has increased in Brazil and ageing, as a biological and psychosocial phenomenon, is a phase of attention of professionals and researchers. Although there are prejudices about sexuality in aging, many elderly people have an active sexual life and are vulnerable to sexually transmitted infections. This documentary research conducted a literature review on sexual health and aging, selecting 68 articles for content analysis. The articles were found between 2001 and 2018, most of them in 2015 and in the areas of Nursing, Collective Health and Gerontology. The results were described in the categories: (A) Violence against elderly; (B) Diseases and physical problems in aging; (C) Sexual health and sexuality; (D) Experiences of elderly with HIV/AIDS. (E) HIV/AIDS and elderly: vulnerability and prevention. The need for sex education to decrease the level of vulnerability of older people to sexual health is concluded.

KEYWORDS: Aging. Sexual Health. Sexual Education.

\section{Introdução}

Ao longo do tempo as sociedades foram atribuindo diferentes significações à velhice, por isso não é possível compreendê-la meramente na sua dimensão biológica, mas sim como um fenômeno histórico, social e cultural (AZEVEDO, 2005). A expectativa de vida no Brasil é de aproximadamente 75 anos, assim como ocorre nas Américas, isto é, a população idosa tem aumentado rapidamente nas últimas décadas, incentivando pesquisadores (as) e profissionais a planejarem ações que promovam a qualidade vida durante essa fase do desenvolvimento humano.

Embora o envelhecimento não seja um fenômeno restrito à população brasileira, o Brasil apresenta uma mudança importante no seu desenho demográfico. Dos anos de 2004 a 2014, o grupo etário que mais apontou crescimento foi das pessoas com mais de 60 anos: um processo conhecido como "transição da estrutura etária" evidenciando a experiência da longevidade, o que tornou necessária a implementação de uma política nacional dirigida aos idosos, seguindo as diretrizes de organizações internacionais sobre programas sociais e assistenciais para essa população (FERNANDES; SOARES, 2012).

No Brasil foi em 1994 a instituição de uma política nacional para os idosos visando garantir o princípio fundamental de que "o idoso é um sujeito de direitos e deve ser atendido de maneira diferenciada em cada uma das suas necessidades físicas, sociais, econômicas e políticas" (CAMARANO; PASINATO, 2004, p. 269). Para a coordenação e gestão dessa política foi designada a Secretaria de Assistência Social do Ministério da Previdência e Assistência Social (MPAS). Outro marco importante foi a aprovação da Política Nacional de Assistência Social (PNAS), em 2004, com sua posterior regulação, em 2005, pelo Sistema 
Único de Assistência Social (Suas), que estabelece um pacto federativo para a operacionalização da PNAS.

O Estatuto do Idoso publicado na Lei $n^{\circ} 10.741$ (BRASIL, 2013) regulamenta os direitos assegurados a todos os cidadãos a partir dos 60 anos de idade. Nesse estatuto há menção ao amparo ao direito de atenção integral à saúde, por intermédio do Sistema Único de Saúde (SUS). Garante o acesso universal e igualitário para prevenção, promoção e proteção, bem como a recuperação da saúde, estabelecendo o atendimento preferencial à pessoa idosa, dentre outras ações. A Política Nacional de Saúde da Pessoa Idosa - PNSPI (BRASIL, 2006) tem como finalidade primordial a recuperação, manutenção e promoção da autonomia e da independência da pessoa idosa, direcionando medidas coletivas e individuais de saúde para esse fim, em consonância com os princípios e diretrizes do Sistema Único de Saúde.

Além das políticas públicas em 2005 há um marco teórico que integra a abordagem multidimensional do envelhecimento e que possui a chancela da Organização Mundial de Saúde (OMS), que adotou o documento "Envelhecimento ativo: um marco para elaboração de políticas". Este documento apresenta os principais desafios a serem enfrentados no mundo, relacionados ao envelhecimento da população, e destaca o fato de que a saúde só pode ser criada e mantida com a participação de vários setores (VERAS, 2009). O envelhecimento ativo é "o processo de otimização das oportunidades de saúde, participação e segurança, com o objetivo de melhorar a qualidade de vida à medida que as pessoas ficam mais velhas" (OMS, 2005, p. $14)$.

Um dos aspectos da vida, importante na qualidade de vida, é a sexualidade. Como um tema ainda polêmico, a sexualidade torna-se ainda mais complexa quando se baseia em vários preconceitos relacionados à vida sexual em idades mais avançadas, apesar do reconhecimento científico de que a sexualidade pode ser vivenciada por todos, independentemente da cronologia (PASCUAL, 2002; VERAS, 2009).

Para compreender o comportamento sexual do idoso há que se olhar para os costumes sexuais construídos em sociedade. As dificuldades sexuais vivenciadas por alguns idosos hoje, pode estar relacionada não só a um fator isolado e sim por consequências e resultados de toda uma história, regulada por códigos e padrões sócio-normativos dentro da sociedade (PASCUAL, 2002). Estudos têm divulgado que os idosos são pessoas sexuadas (ALENCAR; MARQUES; LEAL; VIEIRA, 2016; NETTO-MARTINS, 2012) e apesar de possíveis dificuldades oriundas do envelhecimento na resposta sexual a prática sexual na idade avançada não só é possível, como é desejável. Alguns autores discutem que a dessexualização no 
envelhecimento não é cronológica é uma questão social (GRANDIM et al., 2007; LINHARES et al., 2008).

Não é a atividade sexual que torna as pessoas vulneráveis às IST e ao HIV/Aids, mas as práticas sexuais que são realizadas de forma desprotegidas e este é um pressuposto válido para todas as idades. No entanto, convém ressaltar que o profissional de saúde deve estar atento para as queixas específicas das pessoas idosas, considerando que são seres sexuados e vulneráveis às infecções sexualmente transmissíveis e outras dificuldades no campo amoroso e sexual (SANTOS; ASSIS, 2011; RODRIGUES; PRAÇA, 2010).

A vivência da sexualidade, assim como em qualquer fase da vida, precisa ser garantida em saúde sexual. Pesquisas, campanhas de prevenção, políticas públicas e serviços especializados tendem a desconsiderar essa parcela da população, quando se trata de sexualidade e, só recentemente, diante dos avanços da medicina que auxiliaram a vida sexual de mulheres e homens idosos (uso de hormônios, medicamentos para ereção peniana, etc.) é que os serviços de saúde têm recebido mais frequentemente essa população. Junto com a vida sexual mais frequente, registrou-se um aumento significativo de idosos contaminados por infecções sexualmente transmissíveis, como a Aids, despertando o interesse de estudiosos na área da saúde sexual e envelhecimento.

O fenômeno dos riscos à saúde sexual no envelhecimento ainda não é tão conhecido pela população em geral ou mesmo pelos profissionais da saúde que deveriam acolher essa demanda. Neste sentido, seria importante um levantamento geral sobre a literatura e o que nela se caracteriza no interesse de pesquisadores (as) sobre essa temática, considerando as pessoas idosas sexualmente ativas, vulneráveis ou com HIV/Aids e outras infecções sexualmente transmissíveis. Diante do exposto, propomos este estudo para investigar o que diz a literatura sobre saúde sexual e envelhecimento.

\section{Método}

Esta pesquisa tem abordagem qualitativa-exploratória, tipo estudo documental (SPATA, 2005), caracterizada como um estudo de revisão sistemática da literatura que é uma modalidade de pesquisa que busca identificar, analisar e apresentar estudos anteriores sobre determinado problema de pesquisa.

Passos comuns foram realizados, tais como: localizar os documentos (artigos), selecioná-los diante de critérios de inclusão e de exclusão, analisar a partir de propósitos prévios e interpretá-los à luz da teoria. No nosso caso, optamos pela seleção dos artigos em uma base 
de dados acessível e geral: scielo.org ${ }^{3}$ que priorizasse os estudos brasileiros, pois nosso interesse posterior era pensar nas políticas públicas. As variáveis estudadas foram "saúde sexual e envelhecimento" e os descritores utilizados foram o cruzamento de: "saúde sexual" e "HIV" com "idoso", "envelhecimento" e "velhice". Todos os 114 artigos que apareceram foram selecionados e depois, excluíram-se os repetidos. A amostra final, a partir do objetivo do eixo resultou em 68 artigos.

Para a análise de dados, os procedimentos que adotamos foi realizar uma leitura atenta de cada um dos artigos com extração temática e conceitual, para posterior organização das categorias temáticas emergentes, a partir da técnica de análise de conteúdo proposta por Bardin (2011).

\section{Resultados}

\section{Caracterização geral dos artigos}

Os artigos encontraram-se entre os anos de 2001 a 2018, sendo a maior concentração deles no ano de 2015. A Figura 1 mostra a distribuição dos artigos da amostra final, ao longo do período em anos.

Figura 1 - Distribuição dos 68 artigos anualmente

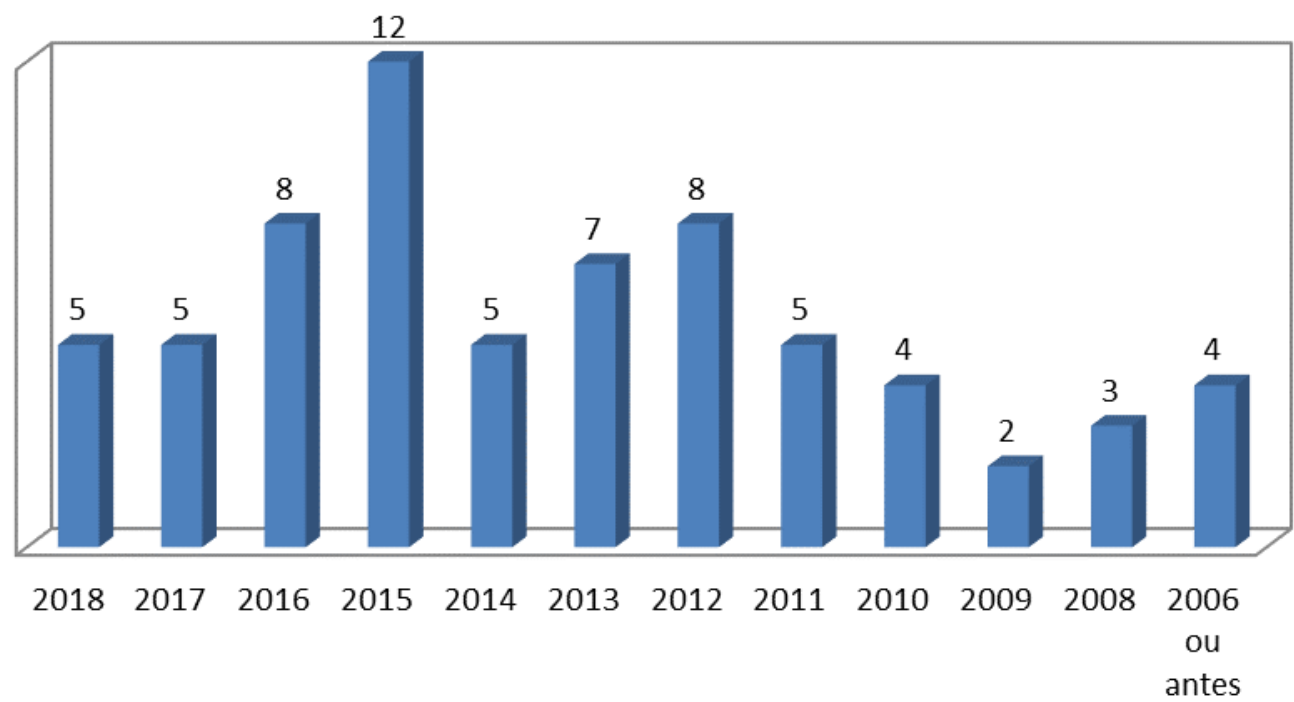

Fonte: Elaborado pelos autores

${ }^{3}$ Disponível em: http://www.scielo.org/php/index.php. Acesso em: 10 set. 2020.

RIAEE - Revista Ibero-Americana de Estudos em Educação, Araraquara, v. 15, n. esp. 4, p. 2699-2712, dez., 2020. e-ISSN: $1982-5587$ 
$\mathrm{Na}$ última década o número de artigos está aumentando, tendo sido expressivo a quantidade em 2015 (=12). Antes de 2006, registramos apenas um artigo a cada ano, sendo um em 2006, um em 2004, um em 2003 e outro em 2001. Grande parte dos artigos foi publicado nas seguintes revistas: "Revista Brasileira de Geriatria e Gerontologia" (n=14), "Revista Brasileira de Enfermagem" (n=7) e "Cadernos de Saúde Pública" (n=6).

As áreas das ciências das revistas foram localizadas a partir da consulta da Revista que é associada a um departamento, universidade ou associação. (Figura 2).

Figura 2 - Distribuição dos artigos pelas diferentes áreas da Ciência

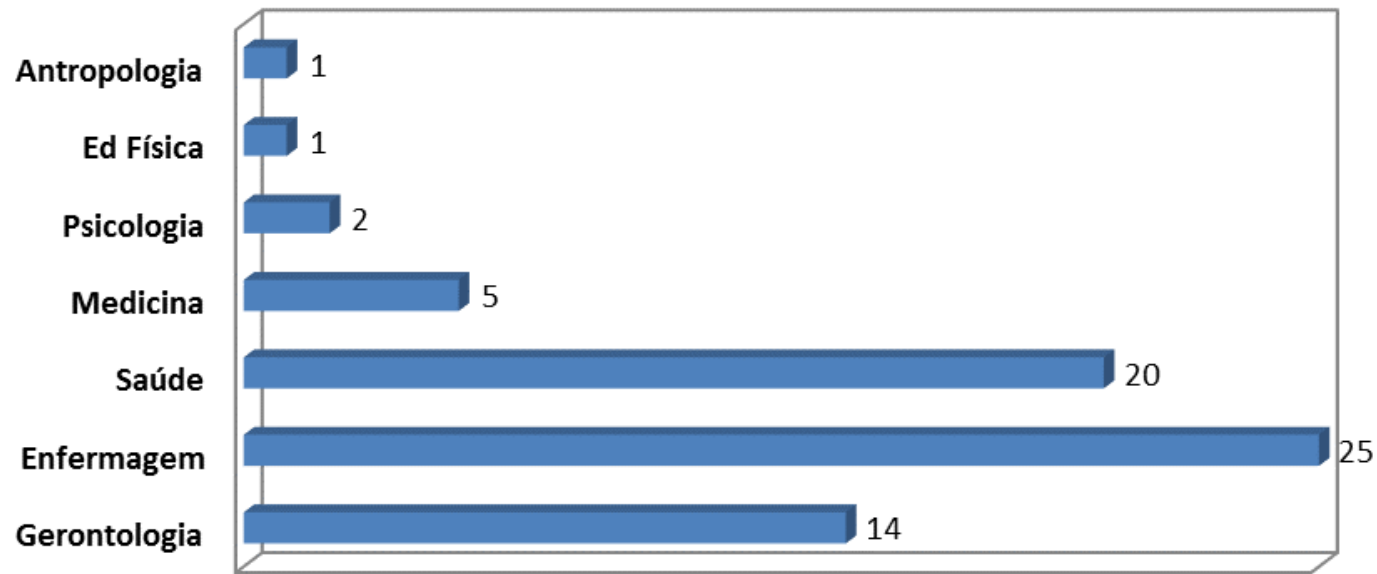

Fonte: Elaborado pelos autores

Assim, quanto à área da ciência, considerando o periódico científico publicado, temos que a maioria dos artigos concentra-se na área da Enfermagem, seguido da Saúde e Gerontologia. Áreas afins foram poucas, tais como "Educação física”, "Antropologia" e a "Psicologia" com apenas duas publicações, o que é bastante limitado.

\section{Análise temática dos artigos}

Os 68 artigos foram distribuídos em cinco categorias envolvendo as temáticas específicas dentro do grande tema que é a saúde sexual no envelhecimento, tal como aparece no Quadro 1. 
Quadro 1 - Distribuição e descrição das categorias temáticas emergentes

\begin{tabular}{|c|c|c|}
\hline Categorias Temáticas & Descrição & $\begin{array}{c}\text { No de artigos } \\
(n=68)\end{array}$ \\
\hline $\begin{array}{c}\text { (A)Violência contra } \\
\text { idosos (as) }\end{array}$ & $\begin{array}{l}\text { Reúne artigos que abordam questões sobre violências físicas, sexuais, } \\
\text { psicológicas, financeiras, etc. envolvendo a pessoa no envelhecimento } \\
\text { ou a relação com questões de saúde e gênero. }\end{array}$ & 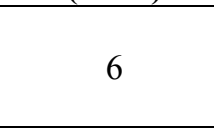 \\
\hline $\begin{array}{l}\text { (B) Doenças e } \\
\text { problemas físicos no } \\
\text { envelhecimento }\end{array}$ & $\begin{array}{l}\text { Envolve artigos que apontam temas específicos sobre problemas de } \\
\text { saúde na idade avançada, relacionando com fatores individuais e } \\
\text { sociais. }\end{array}$ & 8 \\
\hline $\begin{array}{l}\text { (C) Saúde sexual e } \\
\text { sexualidade }\end{array}$ & $\begin{array}{l}\text { Artigos que tratam de questões amplas da sexualidade, como } \\
\text { relacionamentos, padrões de beleza, relações de gênero e as } \\
\text { específicas da resposta sexual e das mudanças hormonais próprias do } \\
\text { envelhecimento. }\end{array}$ & 14 \\
\hline $\begin{array}{l}\text { (D) Vivências de } \\
\text { idosos com } \\
\text { HIV/aids: } \\
\text { incidência, dados } \\
\text { epidemiológicos, } \\
\text { diagnóstico e } \\
\text { tratamento } \\
\end{array}$ & $\begin{array}{l}\text { Estudos que apresentam dados sobre as incidências de HIV/Aids em } \\
\text { pessoas idosas, bem como o perfil e outras características em } \\
\text { diferentes contextos. Além disso, os artigos apresentam informações } \\
\text { obtidas com as pessoas idosas que já tem HIV/Aids sobre o } \\
\text { diagnóstico recebido, adesão e modos de tratamento, hábitos, } \\
\text { dificuldades e enfrentamentos diante das necessidades de cuidados } \\
\text { após a doença e implicações disso para a garantia de qualidade de vida. }\end{array}$ & 20 \\
\hline $\begin{array}{c}\text { (E) HIV/aids e } \\
\text { idosos: } \\
\text { Vulnerabilidade e } \\
\text { prevenção }\end{array}$ & $\begin{array}{l}\text { Estudos que apresentam dados obtidos junto a profissionais da saúde } \\
\text { e/ou cuidadores de idosos que têm HIV/Aids ou discutem níveis de } \\
\text { informações, conhecimento sobre sexualidade, fatores na vida que } \\
\text { levam a vulnerabilidade de idosos diante do contágio de HIV/Aids. } \\
\text { Além disso, os artigos citam estratégias de prevenção em saúde sexual } \\
\text { voltadas a população idosa. }\end{array}$ & 20 \\
\hline
\end{tabular}

Fonte: Elaborado pelos autores

$\mathrm{Na}$ Categoria A- "Violência contra idosos (as)", observamos que os estudos foram realizados a partir de dados registrados em centros de saúde e em sistemas de informações e não diretamente entrevistando os próprios idosos. Os artigos denunciam os altos índices de violência contra os idosos, sendo a física a mais comum, acometidas contra homens idosos e por agressores externos à família, seguida da sexual e psicológica que ocorrem mais com as idosas mulheres, por pessoas conhecidas.

A Categoria B- "Doenças e problemas físicos no envelhecimento" apresentou artigos que descreveram aspectos fisiológicos relacionados ao envelhecimento, apontando fatores importantes que possam se relacionar com a saúde e a qualidade de vida, na idade avançada. Doenças como tuberculose, hipotermia, assim como sintomas de transtornos de humor (depressão), foram pesquisados considerando fatores individuais de proteção, como atividade física, relações sexuais, assim como fatores sociais, como condições econômicas favoráveis e políticas públicas eficazes.

No campo da sexualidade (Categoria C- "Saúde sexual e sexualidade"), os temas abordados sobre a resposta sexual foram sobre o uso de hormônios e medicalização e os interesses mercadológicos de seus usos. Fatores orgânicos, como dor crônica ou hábitos saudáveis foram destacados como possíveis variáveis que interferem na vida sexual satisfatória 
ou não. Mas, sobretudo, fatores psicossociais são apresentados em vários estudos - mitos e preconceitos, falta de parceiro, desinformações, padrões de beleza, feminilidade e masculinidade, como importantes influências sociais e culturais no exercício da sexualidade no envelhecimento.

Muitos artigos concluem a necessidade de atenção por parte de profissionais da saúde sobre a sexualidade no envelhecimento, sobretudo, quanto à vulnerabilidade dessa população diante dos riscos de doenças. Dois estudos de revisão e um texto teórico complementam as informações obtidas pela análise dos artigos nesta categoria, ressaltando a importância dos fatores sociais, econômicos, psicológicos, as questões de gênero, a qualidade da saúde, as condições de educação e de esclarecimento quando se analisa a sexualidade de pessoas idosas, em diferentes contextos e condições.

$\mathrm{Na}$ Categoria D- "Vivências de idosos com HIV/aids: incidência, dados epidemiológicos, diagnóstico e tratamento" os artigos convergem, de modo geral, para algumas características. A idade média de obtenção do diagnóstico é entre 60 e 69 anos, embora a contaminação possa ocorrer antes. Os estudos mais antigos mostram uma proporção maior de homens idosos do que mulheres, que ao logo dos anos foi diminuindo significativamente, atingindo atualmente, muitas mulheres. A maioria dos (as) idosos (as) com HIV/Aids tem baixa escolaridade, foram contaminados por relações sexuais, heterossexuais. Os estudos brasileiros mostram essas informações a partir de diferentes estados do país: Distrito Federal, Ceará, Rio Grande do Sul, Rio de Janeiro, Minas Gerais e Rondônia e os dados obtidos mostram uma realidade desde 1998 até os dias de hoje.

Viver com o HIV/Aids e ser idoso (a), implica em somar as condições da soropositividade e de seu tratamento às mudanças fisiológicas comuns no envelhecimento, que aumentam as chances do desenvolvimento de doenças associadas e dificuldades psicossociais. Diante do diagnóstico, é comum que os (as) idosos (as) nem saibam como ocorreu a transmissão e evidenciam representações negativas sobre a Aids que dificultaram a eles e as famílias, aceitar a nova condição. Geralmente o diagnóstico para essa população acaba ocorrendo de modo tardio, porque os próprios profissionais não investigam essa possibilidade e acabam dialogando com eles (as) sobre sexo e prevenção, somente após já terem se contaminado.

A relação entre variáveis que representam fatores de risco, tais como, nível educacional ou outras doenças, ou que representam fatores de proteção, tais como nível educacional elevado, conhecimento e domínio de informações sobre o contágio e a prevenção ou a manutenção de um corpo saudável (atividades físicas, alimentação, etc.) 
foram focos de alguns estudos para avaliar a qualidade de vida de idosos (as) com HIV/Aids. Qualidade de vida essa, afetada por preocupações com o sigilo, com a vida sexual ou com dificuldades financeiras, além de dificuldades de aceitação do diagnóstico ou do uso da medicação. Dificuldades na adesão ao tratamento foram destacadas em função de atraso ou não uso do medicamento, os efeitos colaterais dos medicamentos ou o uso de bebidas alcoólicas; também houve estudos sobre as influências da terapia antirretroviral e da avaliação da capacidade funcional dessas pessoas. Também estudos destacaram os impactos psicossociais que foram relatados pelos idosos e que levam a uma necessidade de reorganização nas suas atitudes sociais e o enfrentamento de preconceitos.

Finalmente, na Categoria E- "HIV/aids e idosos: Vulnerabilidade e prevenção", os estudos evidenciaram a condição de vulnerabilidade da população idosa diante do contágio de IST em geral, sobretudo quanto ao HIV/Aids. Por um lado, as pessoas idosas não têm ou têm pouca informação sobre o tema e não aderem às práticas preventivas e, por outro, há uma invisibilidade da vida sexual ativa por parte dos profissionais da saúde e falta de políticas públicas que invistam em programas de intervenção para prevenção.

Em muitos casos, as pessoas idosas conhecem sobre o HIV/Aids, mas não se reconhecem vulneráveis e não usam o preservativo, mantendo o imaginário que a Aids não os atingem. Mesmo quando há idosos que se reconhecem suscetíveis à infecção, é comum não adotarem as medidas de proteção, como usar o preservativo, muitas vezes, por terem dificuldades para negociar seu uso com parceiros (as), principalmente quando se trata de mulheres idosas.

Também nessa categoria, as concepções e opiniões dos profissionais envolvidos no atendimento da clientela idosa foram registrados nos estudos. Há o reconhecimento da vulnerabilidade dessa população ao contágio de HIV/Aids, mas por serem idosos, quando há o contágio, associam a necessidade de maiores cuidados e identificam os impactos desse diagnóstico na vida deles: isolamento, solidão, medo e vergonha diante do preconceito.

A discussão dos termos incluídos na Classificação Internacional para a Prática da Enfermagem chama a atenção para a preocupação desses profisssionais em nomear com maior precisão os diagnósticos e tratamentos que devem ser direcionados aos idosos, quando se trata da contaminação dessas infecções sexuais. Entretanto, há estudos que mostram que esses profissionais enfatizam o atendimento individualizado e clínico de idosos com HIV/Aids e não ampliam para uma discussão sobre os aspectos sociais envolvidos na vulnerabilidade e que deveriam ser considerados no tratamento. 
Apenas dois artigos referiram-se a propostas de intervenção em educação sexual e levantaram uma questão fundamental no cenário da vulnerabilidade e aumento de índices de HIV/Aids em idosos: a prevenção. Há uma proposta de intervenção com um trabalho em grupo que favorece a divulgação de informações e reflexões sobre a importância das atitudes preventivas e há a descrição de uma cartilha como um material pedagógico que auxiliasse também nesse mesmo propósito.

\section{Discussão}

Os artigos foram localizados no período de duas décadas atrás, sugerindo que o fenômeno da saúde sexual no envelhecimento, principalmente sobre o contágio e a prevenção do HIV/Aids ganhou destaque entre os (as) pesquisadores (as), na medida em que os índices das taxas de contaminação foram sendo mais notificados (BRASIL, 2017). Em 2015 há um aumento expressivo do número de artigos e nossa hipótese é que os dados epidemiológicos que entre 2006 e 2014 mostraram um aumento expressivo de contaminação por HIV/Aids em idosos e uma diminuição entre adultos.

A maior parte dos artigos foi publicado em revistas cujas áreas do conhecimento eram específicas do envelhecimento (Geriatria e Gerontologia) ou do campo da saúde (Enfermagem e Saúde pública), ressaltando que a educação sexual ainda é um campo restrito das ciências humanas, pelo menos no que se refere à prevenção da saúde sexual de idosos. Maia e Ribeiro (2011) lembram que os projetos de intervenção em educação sexual devem considerar todas as etapas do desenvolvimento humano e não somente a adolescência e a idade adulta.

A busca nesta revisão da literatura focalizou o tema da saúde sexual no envelhecimento e é importante ressaltar que neste eixo de investigação outros temas foram desvelados: doenças, situações de violência e sexualidade. Doenças são comuns no envelhecimento, mas poucos estudos relacionam o adoecimento à saúde sexual ou falta dela (possíveis dificuldades na vida afetiva e sexual). Sobre sexualidade e envelhecimento, sabemos que a literatura tem expressiva contribuição, pois há livros e artigos discutindo, cada vez mais, a sexualidade inerente ao ser humano existente em toda a vida (ALENCAR et. al., 2016; GRANDIM et. al., 2007; LINHARES et. al., 2008; NETTO-MARTINS, 2012).

No caso de relacionar a sexualidade à saúde sexual, os artigos encontrados foram mais restritos e consideraram além dos fatores biológicos (alterações na resposta sexual), também os fatores psicológicos e sociais, diante de modelos definidores de normalidade, como estética, sensualidade, vigor sexual etc (MAIA, 2008). Além disso, uma discussão atual foi questionar 
o uso de hormônios e medicamentos que, por um lado contribuem no exercício da prática sexual, mas por outro, medicalizam o sujeito no sentido de administrar sua subjetividade (CARVALHO; RODRIGUES; COSTA; ANDRADE, 2015).

A temática do HIV entre idosos foi predominante na relação entre saúde e envelhecimento ( $\mathrm{n}=40$ artigos). Um grupo pesquisas descreve o perfil dessas pessoas, incidência da contaminação e reações ao diagnóstico e ao tratamento. No contexto da atenção secundária foram os profissionais da saúde, como médicos e enfermeiros, que tiveram acesso a essa população para obtenção desses dados e, em alguns artigos, reconheceram a importância dos sentimentos, temores, dificuldades dessa população para lidarem com essa situação em suas vidas, sem que em nenhum estudo com profissional psicólogo tenha sido mencionado. Bezerra et al. (2015) e Santos e Assis (2011) defendem que é preciso considerar as dificuldades subjetivas e sociais relacionadas à vivência da sexualidade no envelhecimento uma vez que o processo de adoecimento por HIV/aids em idosos está relacionado a preconceitos, estigmas e discriminação.

Outro grupo de estudos ressalta a vulnerabilidade das pessoas idosas quanto ao contagio de HIV/Aids, seja pelo pouco conhecimento das informações necessárias para se protegerem, seja pela invisibilidade de suas vidas sexuais e situações de riscos, refletidos nos preconceitos dos profissionais, dos familiares e deles próprios (LAROQUE et al., 2011).

Silva et al. (2015, p. 821) lembram que o aumento do número de idosos infectados por HIV/Aids traz um desafio "às políticas públicas e à população em geral no que concerne à necessidade de discutir sexualidade, práticas sexuais no processo de envelhecimento e o impacto dessas questões na promoção de saúde da pessoa idosa”. Dos 68 artigos, apenas dois mencionavam propostas de intervenção visando a prevenção, sugerindo ainda a propagação do mito da assexualidade dessa população e pouca preocupação com campanhas e projetos voltados exclusivamente ao público idoso (ALENCAR et al., 2016; NETTO-MARTINS, 2012; PASCUAL, 2002; VERAS, 2009).

Além disso, também apontam a pouca experiência e formação dos profissionais de saúde envolvidos na atenção primária das pessoas idosas, bem como as precárias políticas públicas de prevenção à saúde sexual nesta fase da vida humana (FERNANDES; SOARES, 2012; SILVA et al., 2015). Ou seja, a análise da literatura indica a necessidade de haver propostas de educação sexual e preventiva em saúde sexual diretamente para as pessoas no envelhecimento, tal como defendem Lazzarotto et al. (2013).

A literatura converge para a necessidade de os profissionais da saúde reconhecerem que os idosos são sexualmente ativos, são vulneráveis ao contágio de IST, dando atenção às 
suas particularidades tanto no atendimento, quando no planejamento e implementação de intervenções (SANTOS; ASSIS, 2011; SILVA et al., 2015).

\section{Considerações}

A revisão da literatura é um procedimento metodológico importante para um levantamento inicial e mapeamento de um fenômeno que, no Brasil, ainda é pouco acessado nas áreas das ciências humanas e sociais, mas já bastante notificado no campo da saúde.

Diante das discussões é preciso não perder de vista a importância do trabalho em equipe interdisciplinar: psicólogos, educadores, médicos, enfermeiros, etc. em todo o processo integral de cuidados com os idosos. Os dados encontrados contribuem para que profissionais da educação sexual tenham conhecimento de elementos importantes para a elaboração e a implementação de propostas de intervenção que possibilitem diminuir o nível de vulnerabilidade de pessoas mais velhas quanto a saúde sexual.

Conclui-se que ainda são necessários esforços para garantir o direito ao exercício da sexualidade no envelhecimento, atendendo as necessidades das pessoas mais velhas nas propostas preventivas, no momento do diagnóstico, no oferecimento e na manutenção do tratamento e no acolhimento de possíveis desdobramentos psicossociais da experiência de ser contaminado por HIV/Aids, para si mesmos, para seus pares e familiares e para a sociedade.

\section{REFERÊNCIAS}

ALENCAR, D.; MARQUES, A. P. O.; LEAL, M. C. C.; VIEIRA, J. C. M. Exercício da sexualidade em pessoas idosas e os fatores relacionados. Rev. bras. geriatr. Gerontol, Rio de Janeiro, v. 19, n. 5, p. 861-869, 2016.

AZEVEDO, A. L. A velhice e seus processos sócio-históricos. Lisboa: Argumento, 2001.

BARDIN, L. Análise de conteúdo. 5. ed. Trad. Luis Antero Reto e Augusto Pinheiro. Lisboa: Edições 70, 2011.

BEZERRA, V. P.; SERRA, M. A. P.; CABRAL, I. P. P.; MOREIRA, M. A. S. P.; ALMEIDA, S. A.; PATRÍCIO, A. C. F. A. Preventive practices in the elderly and vulnerability to HIV. Rev Gaúcha Enferm, Porto Alegre, v. 36, n. 4, p. 70-76, 2015.

BRASIL. Ministério da Saúde. Boletim Epidemiológico HIV Aids 2017. Brasília, DF: Ministério da Saúde, 2017. Disponível em: https://central3.to.gov.br/arquivo/387532/. Acesso em: 20 jan. 2019.

BRASIL. Ministério da Saúde. Estatuto do idoso. 3. ed. Brasília, DF: Ministério da Saúde, 2013. Disponível em: http://bvsms.saude.gov.br/bvs/publicacoes/estatuto_idoso_3edicao.pdf. Acesso em: 20 jan. 2019. 
BRASIL. Ministério da Saúde. Portaria n. 2.528 de 19 de outubro de 2006. Aprova a Política Nacional da saúde da pessoa idosa. Brasília, DF, 2006. Disponível em: http://bvsms.saude.gov.br/bvs/saudelegis/gm/2006/prt2528_19_10_2006.html. Acesso em: 20 jan. 2019.

CAMARAnO, A. A.; PASINATO, M. T. O Envelhecimento Populacional na Agenda das Políticas Públicas. In: CAMARANO, A. A. (Org.) Os novos idosos brasileiros: muito além dos 60? Rio de Janeiro: Instituto de Pesquisa Econômica e Aplicada (Ipea), 2004. p. 253-292. Disponível em: http://www.ipea.gov.br/portal/images/stories/PDFs/livros/Arq_29_Livro_Completo.pdf. Acesso em: 20 jan. 2019.

CARVALHO, S. R.; RODRIGUES, C. O.; COSTA, F. D.; ANDRADE, M. S. Medicalização: uma crítica (im) pertinente? Physis Revista de Saúde Coletiva, Rio de Janeiro, v. 25, n. 4, p. 1251-1269, 2015.

FERNANDES, M. T. O.; SOARES, S. M. O desenvolvimento de políticas públicas de atenção ao idoso no Brasil. Rev. esc. enferm. USP, São Paulo, v. 46, n. 6, p. 1494-1502, 2012.

GRADIM, C. V. C.; SOUSA, A. M.; LOBO, J. M. A prática sexual e o envelhecimento. Cogitare Enfermagem, Curitiba, v. 12, n. 2, nov. 2007. Disponível em:

https://revistas.ufpr.br/cogitare/article/view/9826/6737. Acesso em: 20 jan. 2019.

LAROQUE, M. F.; AFFELDT, A. B.; CARDOSO, D. H.; SOUZA, G. L.; SANTANA, M. G.; LANGE, C. Sexualidade do idoso: comportamento para a prevenção de DST/AIDS. Rev. Gaúcha de Enfermagem, Porto Alegre, v. 32, n. 4, p. 774-780, 2011.

LAZZAROTTO, A. R. et al. Oficinas educativas sobre HIV/Aids: uma proposta de intervenção para idosos. Rev. bras. de geriatr e gerontol, Rio de Janeiro, v. 14, n. 4, p. 833-843, 2013.

LINHARES, F. M. P. et al. Percepção de idosos sobre o exercício da sexualidade atendidos no Núcleo de Atenção ao idoso em Recife. Rev. enferm. Herediana, Recife, v. 1, n. 2, p. 93-103, 2008.

MAIA, A. C. B. A Educação Sexual Repressiva: padrões definidores de normalidade. In: SOUZA, C. B. G.; RIBEIRO, P. R. M. (Org.). Sexualidade, Diversidade e Culturas Escolares: contribuições ibero-americanas para estudos de educação, gênero e valores. Araraquara: Laboratório Editorial da FCLar-UNESP; Alcalá de Henares: Universidad de Alcalá, 2008. p. 67-83. (Série Temas em Educação Escolar, n. 9)

MAIA, A. C. B.; RIBEIRO, P. R. M. Educação Sexual: princípios para a ação. Doxa Revista Paulista de Psicologia e Educação, Araraquara, v. 15, n. 1, p. 41-51, 2011.

NETTO-MARTINS, T. C. R. Sexualidade e Envelhecimento na Percepção de Pessoas Idosas. Orientadora: Ana Cláudia Bortolozzi Maia. 2012. 140 f. Dissertação (Mestrado em Psicologia) Universidade Estadual Paulista Júlio de Mesquita Filho, Bauru, 2012.

ORGANIZAÇÃO MUNDIAL DA SAÚDE (OMS). Envelhecimento ativo: uma política de saúde. Brasília: Organização Pan-Americana de Saúde, 2005. 61 p. Disponível em: http://bvsms.saude.gov.br/bvs/publicacoes/envelhecimento_ativo.pdf. Acesso em: 20 jan. 2019.

PASCUAL, C. P. A sexualidade do idoso vista com novo olhar. São Paulo: Loyola, 2002.

RODRIGUES, D. A. L.; PRACA, N. S. Mulheres com idade igual ou superior a 50 anos: ações preventivas da infecção pelo HIV. Rev. Gaúcha Enferm, Porto Alegre, v. 31, n. 2, p. 321-327, 2010.

RIAEE - Revista Ibero-Americana de Estudos em Educação, Araraquara, v. 15, n. esp. 4, p. 2699-2712, dez., 2020. e-ISSN: 1982-5587 
SANTOS, A. F. M.; ASSIS, M. Vulnerabilidade das idosas ao HIV/AIDS: despertar das políticas públicas e profissionais de saúde no contexto da atenção integral: revisão de literatura. Rev. bras. geriatr. gerontol., Rio de Janeiro, v. 14, n. 1, p. 147-157, 2011.

SILVA, L. C.; FELICIO, E. E. A. A.; CASSÉTE, J. B.; SOARES, L. A.; MORAIS, R. A.; PRADO, T. S.; GUIMARÃES, D. A. Psychosocial impact of HIV/aids diagnosis on elderly persons receiving care from a public healthcare service. Rev. bras. geriatr. gerontol., Rio de Janeiro, v. 18, n. 4, p. 821-833, 2015.

SPATA, A. Métodos de pesquisa: ciências do comportamento e diversidade humana. Rio de Janeiro: LTC, 2005.

VERAS, R. P. Envelhecimento Populacional Contemporâneo: demandas, desafios e inovações. Rev. Saúde Pública, Rio de Janeiro, v. 43, p. 548-554, 2009.

\section{Como referenciar este artigo}

BORTOLOZZI, A. C.; NETTO, T. C. R. Saúde sexual e envelhecimento: revisão da literatura e apontamentos para a educação sexual. Revista Ibero-Americana de Estudos em Educação, Araraquara, v. 15, n. esp. 4, p. 2699-2712, dez., 2020. e-ISSN: 1982-5587. DOI: https://doi.org/10.21723/riaee.v15iesp4.14516

Submetido em: 10/09/2019

Revisões requeridas: $10 / 01 / 2020$

Aprovado em: 30/04/2020

Publicado em: 01/12/2020 